\title{
Front Matter: Volume 6506
}

, "Front Matter: Volume 6506," Proc. SPIE 6506, Multimedia Content Access: Algorithms and Systems, 650601 (29 January 2007); doi: 10.1117/12.724848

SPIE. Event: Electronic Imaging 2007, 2007, San Jose, CA, United States 


\title{
Multimedia Content Access: Algorithms and Systems
}

\author{
Alan Hanjalic \\ Raimondo Schettini \\ Nicu Sebe \\ Chairs/Editors
}

31 January-1 February 2007

San Jose, California, USA

Sponsored and Published by

IS\&T-The Society of Imaging Science and Technology

SPIE-The International Society for Optical Engineering 
The papers included in this volume were part of the technical conference cited on the cover and title page. Papers were selected and subject to review by the editors and conference program committee. Some conference presentations may not be available for publication. The papers published in these proceedings reflect the work and thoughts of the authors and are published herein as submitted. The publishers are not responsible for the validity of the information or for any outcomes resulting from reliance thereon.

Please use the following format to cite material from this book:

Author(s), "Title of Paper," in Multimedia Content Access: Algorithms and Systems, edited by Alan Hanjalic, Raimondo Schettini, Nicu Sebe, Proceedings of SPIE-IS\&T Electronic Imaging, SPIE Vol. 6506, Article CID Number (2007).

ISSN 0277-786X

ISBN 9780819466198

Copublished by

SPIE-The International Society for Optical Engineering

P.O. Box 10, Bellingham, Washington 98227-0010 USA

Telephone 1 360/676-3290 (Pacific Time) · Fax 1 360/647-1445

http://www.spie.org

and

IS\& T-The Society for Imaging Science and Technology

7003 Kilworth Lane, Springfield, Virginia, 22151 USA

Telephone 1 703/642-9090 (Eastern Time) · Fax 1 703/642-9094

http://www.imaging.org

Copyright (@) 2007, The Society of Photo-Optical Instrumentation Engineers and The Society for Imaging Science and Technology.

Copying of material in this book for internal or personal use, or for the internal or personal use of specific clients, beyond the fair use provisions granted by the U.S. Copyright Law is authorized by SPIE and IS\&T subject to payment of copying fees. The Transactional Reporting Service base fee for this volume is $\$ 15.00$ per article (or portion thereof), which should be paid directly to the Copyright Clearance Center (CCC), 222 Rosewood Drive, Danvers, MA 01923. Payment may also be made electronically through CCC Online at http://www.copyright.com. Other copying for republication, resale, advertising or promotion, or any form of systematic or multiple reproduction of any material in this book is prohibited except with permission in writing from the publisher. The CCC fee code is 0277-786X/07/\$15.00.

Printed in the United States of America. 


\section{Contents}

vii Conference Committee

SESSION 1 IMAGE ANALYSIS AND RETRIEVAL

650602 A model-based conceptual clustering of moving objects in video surveillance [6506-01] J. Lee, P. Rajauria, S. K. Shah, Univ. of Bridgeport (USA)

650603 Image watermarking based on a color quantization process [6506-02]

J.-B. Thomas, G. Chareyron, A. Trémeau, Univ. Jean Monnet (France)

650604 Search and retrieval of medical images for improved diagnosis of neurodegenerative diseases [6506-03]

A. Ekin, R. Jasinschi, Philips Research (Netherlands); E. Turan, Sabanci Univ. (Turkey);

R. Engbers, Philips Research (Netherlands); J. van der Grond, M. A. van Buchem, Leiden

Univ. Medical Ctr. (Netherlands)

\section{SESSION 2 CONTENT ANALYSIS-BASED BROWSING}

650605 Assessment of end-user response to sports highlights extraction for personal video recorders (Invited Paper) [6506-04]

H. Shubin, A. Divakaran, K. Wittenburg, K. A. Peker, R. Radhakrishnan, Mitsubishi Electric

Research Labs. (USA)

650606 Examining user interactions with video retrieval systems (Invited Paper) [6506-05]

M. G. Christel, Carnegie Mellon Univ. (USA)

650607 Automatic and user-centric approaches to video summary evaluation (Invited Paper) [6506-06]

C. M. Taskiran, F. Bentley, Motorola Labs. (USA)

Pagination: Proceedings of SPIE follow an e-First publication model, with papers published first online and then in print and on CD-ROM. Papers are published as they are submitted and meet publication criteria. A unique, consistent, permanent citation identifier (CID) number is assigned to each article at the time of the first publication. Utilization of CIDs allows articles to be fully citable as soon they are published online, and connects the same identifier to all online, print, and electronic versions of the publication.

SPIE uses a six-digit CID article numbering system in which:

- The first four digits correspond to the SPIE volume number.

- The last two digits indicate publication order within the volume using a Base 36 numbering system employing both numerals and letters. The CID number appears on each page of the manuscript. The complete citation is used on the first page, and an abbreviated version on subsequent pages. 
650608 Efficient re-indexing of automatically annotated image collections using keyword combination [6506-07]

A. Yavlinsky, S. Rüger, Imperial College London (United Kingdom)

650609 Video to the rescue of audio: shot boundary assisted speaker change detection [6506-08]

A. Samour, M. Karaman, L. Goldmann, T. Sikora, Technical Univ. of Berlin (Germany)

65060A A trajectory based video segmentation for surveillance applications [6506-09]

N. M. Thomas, N. Canagarajah, Univ. of Bristol (United Kingdom)

\section{SESSION 4 APPLICATIONS I}

65060B Knowledge discovery for better photographs [6506-10]

J. Yen, Toshiba America (USA); P. WU, D. Tretter, Hewlett Packard Labs. (USA)

65060C Organising a daily visual diary using multifeature clustering [6506-12]

C. Ó Conaire, N. E. O'Connor, A. F. Smeaton, G. J. F. Jones, Dublin City Univ. (Ireland)

SESSION 5 APPLICATIONS II

65060D Recognizing persons in images by learning from videos [6506-13]

E. Hörster, J. Lux, R. Lienhart, Univ. of Augsburg (Germany)

65060E Storage format for personalized broadcasting content consumption [6506-14]

S. H. Jin, J.-S. Jang, H.-S. Min, Y. M. Ro, Information and Communications Univ. (South Korea); H. Y. Kim, Electronics and Telecommunications Research Institute (South Korea)

65060F A unified and efficient framework for court-net sports video analysis using 3D camera modeling [6506-15]

J. Han, Univ. of Technology Eindhoven (Netherlands); P. H. N. de With, Univ. of Technology

Eindhoven (Netherlands) and LogicaCMG (Netherlands)

\section{SESSION $6 \quad$ BIOINFORMATICS}

$65060 \mathrm{G}$ Ontology driven image search engine (Invited Paper) [6506-16]

Y. Bei, J. Dmitrieva, M. Belmamoune, F. J. Verbeek, Leiden Univ. (Netherlands)

$65060 \mathrm{H}$ Adaptation of video game UVW mapping to 3D visualization of gene expression patterns (Invited Paper) [6506-17]

P. D. Vize, V. E. Gerth, Univ. of Calgary (Canada)

65060 Classification of yeast cells from image features to evaluate pathogen conditions (Invited Paper) [6506-18]

P. van der Putten, L. Bertens, J. Liu, Leiden Univ. (Netherlands); F. Hagen, T. Boekhout, Netherlands Royal Academy of Science (Netherlands); F. J. Verbeek, Leiden Univ. (Netherlands) 
65060J Analysis of unstructured video based on camera motion [6506-20]

G. Abdollahian, E. J. Delp, Purdue Univ. (USA)

65060K Dialog detection in narrative video by shot and face analysis [6506-21]

B. Kroon, Philips Research Europe (Netherlands) and Delft Univ. of Technology

(Netherlands); J. Nesvadba, Philips Research Europe (Netherlands); A. Hanjalic, Delft Univ. of Technology (Netherlands)

65060L Edit while watching: home video editing made easy [6506-22]

M. Campanella, H. Weda, M. Barbieri, Philips Research Europe (Netherlands)

\section{SESSION $8 \quad$ APPLICATIONS III}

65060M Multi-module human motion analysis from a monocular video [6506-23]

W. Lao, J. Han, Eindhoven Univ. of Technology (Netherlands); P. H. N. de With, Eindhoven Univ. of Technology (Netherlands) and LogicaCMG (Netherlands)

65060N A study on video viewing behavior: application to movie trailer miner [6506-24]

S. Mongy, C. Djeraba, LIFL, CNRS, Univ. of Lille 1 (France)

650600 ARGOS: French evaluation campaign for benchmarking of video content analysis methods [6506-25]

J. Benois-Pineau, LABRI (France); P. Joly, IRIT (France); E. Kijak, IRISA (France); G. Quénot, CLIPS-IMAG CNRS (France)

65060P Data mining learning bootstrap through semantic thumbnail analysis [6506-26]

S. Battiato, G. M. Farinella, G. Giuffrida, G. Tribulato, Univ. di Catania (Italy)

65060Q A spatiotemporal decomposition strategy for personal home video management [6506-27] H. Yi, UIUC (USA); I. Kozintsev, M. Polito, Y. WU, J.-Y. Bouguet, A. Nefian, C. Dulong, Intel Corp. (USA)

Author Index 
Downloaded From: https://www.spiedigitallibrary.org/conference-proceedings-of-spie on 26 Apr 2023

Terms of Use: https://www.spiedigitallibrary.org/terms-of-use 


\title{
Conference Committee
}

\author{
Symposium Chairs
}

Michael A. Kriss, Consultant (USA)

Robert A. Sprague, Consultant (USA)

Conference Chairs

Alan Hanjalic, Technische Universiteit Delft (Netherlands)

Raimondo Schettini, DISCo/Università degli Studi di Milano-Bicocca (Italy)

Nicu Sebe, Universiteit van Amsterdam (Netherlands)

Conference Cochairs

Edward Y. Chang, Google, Inc. (USA)

Simone Santini, Universidad Autónoma de Madrid (Spain)

Theo Gevers, Universiteit van Amsterdam (Netherlands)

Program Committee

Kiyoharu Aizawa, The University of Tokyo (Japan)

Noboru Babaguchi, Osaka University (Japan)

Nozha Boujemaa, INRIA Rocquencourt (France)

Augusto Celentano, Università Ca' Foscari di Venezia (Italy)

Tsuhan Chen, Carnegie Mellon University (USA)

TatSeng Chua, National University of Singapore (Singapore)

Rita Cucchiara, Università degli Studi di Modena e Reggio Emilia (Italy)

Alberto Del Bimbo, Università degli Studi di Firenze (Italy)

Ajay Divakaran, Mitsubishi Electric Research Laboratories (USA)

Chitra Dorai, IBM Thomas J. Watson Research Center (USA)

Arun Hampapur, IBM Thomas J. Watson Research Center (USA)

Alexander G. Hauptmann, Carnegie Mellon University (USA)

Roger D. Hersch, École Polytechnique Fédérale de Lausanne (Switzerland)

Ebroul Izquierdo, Queen Mary University of London (United Kingdom)

Alejandro Jaimes, Fuji Xerox Company, Ltd. (Japan)

Mohan S. Kankanhalli, National University of Singapore (Singapore)

John R. Kender, Columbia University (USA)

Anil C. Kokaram, The University of Dublin, Trinity College (Ireland)

Clement H. C. Leung, Victoria University of Technology (Australia)

Michael S. Lew, Universiteit Leiden (Netherlands)

Rainer W. Lienhart, Universität Augsburg (Germany)

Kadir A. Peker, Mitsubishi Electric Research Laboratories (USA)

Silvia Pfeiffer, Commonwealth Scientific and Industrial Research

Organisation (Australia)

Alan F. Smeaton, Dublin City University (Ireland) 
John R. Smith, IBM Thomas J. Watson Research Center (USA)

Hari Sundaram, Arizona State University (USA)

Ahmet M. Tekalp, University of Rochester (USA)

Qi Tian, The University of Texas at San Antonio (USA)

Alain Trémeau, Université Jean Monnet Saint-Etienne (France)

Luc J. Van Gool, Katholieke Universiteit Leuven (Belgium) and ETH Zürich (Switzerland)

Svetha Venkatesh, Curtin University of Technology (Australia)

Marcel Worring, Universiteit van Amsterdam (Netherlands)

Lei Zhang, Microsoft Research Asia (China)

\author{
Session Chairs \\ $1 \quad$ Image Analysis and Retrieval \\ Jan Nesvadba, Philips Research Laboratories (Netherlands) \\ 2 Content Analysis-based Browsing \\ Ajay Divakaran, Mitsubishi Electric Research Laboratories (USA) \\ 3 Video Analysis and Retrieval I \\ Ahmet Ekin, Philips Research Laboratories (Netherlands) \\ 4 Applications I \\ Cuneyt M. Taskiran, Motorola, Inc. (USA) \\ 5 Applications II \\ Michael G. Christel, Carnegie Mellon University (USA) \\ 6 Bioinformatics \\ Fons J. Verbeek, Universiteit Leiden (Netherlands) \\ $7 \quad$ Video Analysis and Retrieval II \\ Alan F. Smeaton, Dublin City University (Ireland) \\ 8 Applications III \\ Raimondo Schettini, Università degli Studi di Milano-Bicocca (Italy)
}

